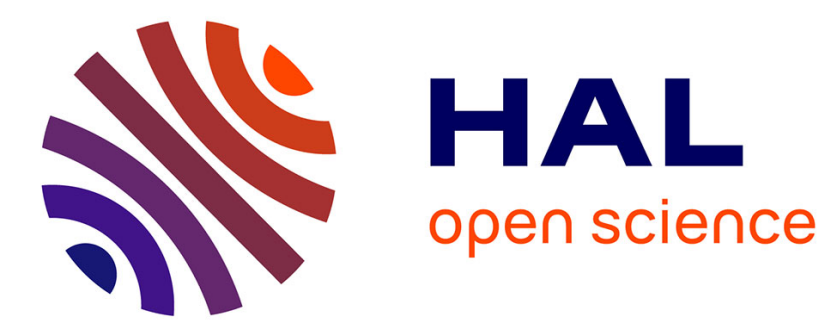

\title{
Nonlinear atomic spectroscopy inside a random porous medium
}

\author{
S Villalba, L Lenci, A Laliotis, D Bloch, A Lezama, H Failache
}

\section{To cite this version:}

S Villalba, L Lenci, A Laliotis, D Bloch, A Lezama, et al.. Nonlinear atomic spectroscopy inside a random porous medium. Latin America Optics and Photonics Conference (LAOP), OSA, Nov 2014, Cancun, Mexico. hal-01090083

\section{HAL Id: hal-01090083 https://hal.science/hal-01090083}

Submitted on 8 Dec 2014

HAL is a multi-disciplinary open access archive for the deposit and dissemination of scientific research documents, whether they are published or not. The documents may come from teaching and research institutions in France or abroad, or from public or private research centers.
L'archive ouverte pluridisciplinaire HAL, est destinée au dépôt et à la diffusion de documents scientifiques de niveau recherche, publiés ou non, émanant des établissements d'enseignement et de recherche français ou étrangers, des laboratoires publics ou privés. 


\title{
Nonlinear atomic spectroscopy in a random porous medium
}

\author{
S. Villalba ${ }^{1}$, L. Lenci ${ }^{1}$, A. Laliotis ${ }^{2}$, D. Bloch ${ }^{2}$, A. Lezama ${ }^{1}$ and H. Failache ${ }^{1, a}$ \\ ${ }^{1}$ Instituto de Física, Facultad de Ingeniería, Universidad de la República, J. Herrera y Reissig 565, \\ 11300 Montevideo, Uruguay \\ ${ }^{2}$ Laboratoire de Physique des Lasers UMR 7538 du CNRS, Université Paris-13, Sorbonne Paris Cité \\ F-93430, Villetaneuse, France \\ ${ }^{a}$ heraclio@fing.edu.uy
}

\begin{abstract}
We studied a novel spectroscopy setup where alkali atoms are infused in random micro-porous glass and the light probing the atoms have a diffuse nature after the propagation in this strong scattering medium.
\end{abstract}

OCIS codes: $300.6210,300.6420$.

\section{Introduction}

An increased interest for the study of disorder is recently observed in the domain of optics and spectroscopy, as can be exemplified by the research on random lasers [1] [2], weak and strong Anderson localization ( [3] and refs. therein), imaging and focussing through or inside highly scattering media [4-7] or the spectroscopy with diffuse light in strongly scattering media like ceramics [8-10] or opals of nano-spheres [11]. In these highly scattering media long effective optical path lengths are observed opening the possibility to develop miniaturized spectroscopic cells.

For few years we have been studying the spectroscopy of dilute rubidium vapor confined to the micrometric interstices of random porous glass where new and interesting problems arise. In this system the light field that probe the atoms deep inside the porous medium is strongly scattered by the medium and have a propagation direction, amplitude and phase strongly randomized. Moreover the volume confining the atoms is also randomized.

In a recent work [12] we have studied the resonant light transmission through this medium in the linear regimen. Surprisingly at low atomic densities, no resonances can be observed. This result is a consequence of the strong spatial randomization of light that introduces the impossibility to distinguish the photons directly coming from the laser from those resonantly scattered by atoms. The photons absorbed by the atoms are almost entirely compensated by fluorescence. At high atomic densities, collisions of excited atoms with the pores wall became more probable introducing a non-radiative decay mechanism and Doppler broadened resonances became then observables. The efficiency of this non-radiative decay was used to consistently determine the characteristic pores dimension. In this work we report recent experiments on non-linear spectroscopy in this singular system.

\section{Sub-Doppler resonances in the back-scattered light}

The atomic spectra detected at the back-scattered light from a porous cell are mainly composed of Doppler broadened lines corresponding to the absorption of $\mathrm{Rb}$ atoms present in the interstitial cavities of the porous sample. However, as shown at Fig.1(b), sub-Doppler resonances are also detected [13]. The presence of cross-over resonances indicates that these features are due to saturated absorption (SA). Moreover, these resonances broaden as the detection angle $\theta$ from the illumination direction is increased. These observations suggest that the incident beam propagates into the porous medium a distance of the order of the scattering mean free path length without significant change in the wave vector direction producing a velocity-selective saturation in the atomic vapor which is probed by radiation scattered backwards in the direction determined by the detector position.

The finite detection solid angle of the diffuse probe radiation determines a spreading of the probe field wave-vector which results in broadening of the SA resonances due to a residual Doppler broadening. At low intensities this broadening mechanism was revealed as the dominant contribution to the linewidth under a small detection angle $\theta \sim$ 0 . Two cells were used with mean pores dimension of $10 \mu \mathrm{m}$ and $100 \mu \mathrm{m}$. For both cells we observed typically a spectral width of $22 \mathrm{MHz}$ for $\theta=0$ and low intensities, using a photodetector with a $0.81 \mathrm{~mm}^{2}$ surface at $10 \mathrm{~cm}$ from the sample. 

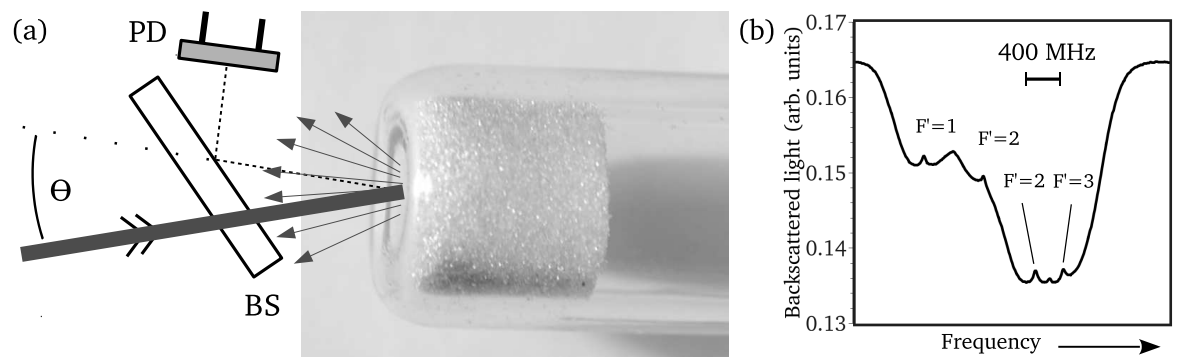

Fig. 1. (a) Scheme of the setup that include a beam splitter (BS) and a photodiode (PD) collecting the back-scattered light at an angle $\theta$ from the incidence beam direction (the photograph shows a porous cell); (b) detected atomic spectra as seen directly at the oscilloscope. The laser is scanned around the ${ }^{87} \mathrm{Rb}\left(\mathrm{F}=2 \rightarrow \mathrm{F}^{\prime}=1,2\right)$ and ${ }^{85} \mathrm{Rb}\left(\mathrm{F}=3 \rightarrow \mathrm{F}^{\prime}=2,3\right)$ transitions.

Transit time broadening was not observed, however a direct manifestation of atomic confinement is observed at the relative size of the cross-over (CO) resonances. As the atoms efficiently contributing to the $\mathrm{CO}$ have relatively large velocities, the $\mathrm{CO}$ amplitudes, compared to the single transition resonances, are much smaller than the amplitude observed at a standard cell. Moreover, CO amplitudes increases with the cell pore size. Atomic confinement is also evidenced at the residual Doppler broadening of resonances as the detection angle $\theta$ is increased. $\mathrm{CO}$ resonances experience a smaller residual Doppler broadening than the transition lines [13].

The experimental setup shown at Fig.1(a) is extremely simple, independent of the porous medium orientation, insensible to its displacements, and the alignment require only a roughly positioning of the detector at the back-scattering direction. Using a $10 \mu \mathrm{m}$ porous cell, $130 \mu \mathrm{W}$ optical power and a beam diameter of $200 \mu \mathrm{m}$, a SA spectra is detected with a typical signal to noise ratio of 50, suggesting its application to the development of miniaturized frequency references.

\section{Incoherent non-linear spectroscopy with the transmitted light}

We have also studied the non-linear pump-probe spectroscopy using two different light fields [14]. These two fields are independently scattered and randomized by the porous medium. Then at any position inside the sample the relative phase of these fields changes stochastically and only effects that do not depend in the relative phase between fields are detected. In the experiments described here a pump field was kept at a fixed frequency while a probe field was frequency scanned over the $\mathrm{D} 1$ line transitions of both $\mathrm{Rb}$ isotopes. The non-linear response of the atomic medium was measured at the light transmitted through the sample detecting the signal dependent on the product of both field intensities. Two different mechanisms contribute to this incoherent non-linear signal. First, saturation of the atomic medium by the pump, that is probed by the other field, and second, optical pumping between the ground levels.

A simplified theoretical model was developed to calculate the spectra line shapes. The model consider first the atoms simultaneously resonant to both light fields that have randomized wave-vectors. This is the only consideration required to explain the large variety of singular spectral shapes experimentally observed. In particular when the pump field is in resonance with a given transition, the spectrum predicted is narrower than the Doppler profile. However, if the pump field is detuned from resonance, the spectrum became considerably broader than de Doppler profile with a sharp decay of the spectrum at its wings. When two nearby transitions are considered, a narrow peak appears (see Fig.2(h) and (k)) that is a direct consequence of the steepness of the decay of the spectra. The model also incorporates the prediction of the relative amplitudes of the spectra using rate equations, that are justified in this case because coherent effect are statistically averaged. The rate equation incorporates as the only free parameter a decay rate $\gamma$ for all atomic levels that accounts in a phenomenological way the interruption of the light-atom interaction due to the collisions with the pores wall. A remarkable feature that should be mentioned is the high sensitivity of the predicted line shapes with the parameter $\gamma$ that is directly linked to the pores dimension.

In the experimental setup, the light beams from two independent lasers were chopped at two different frequencies and sent to the porous sample. A portion of the light intensity transmitted through the sample measured with a pho- 

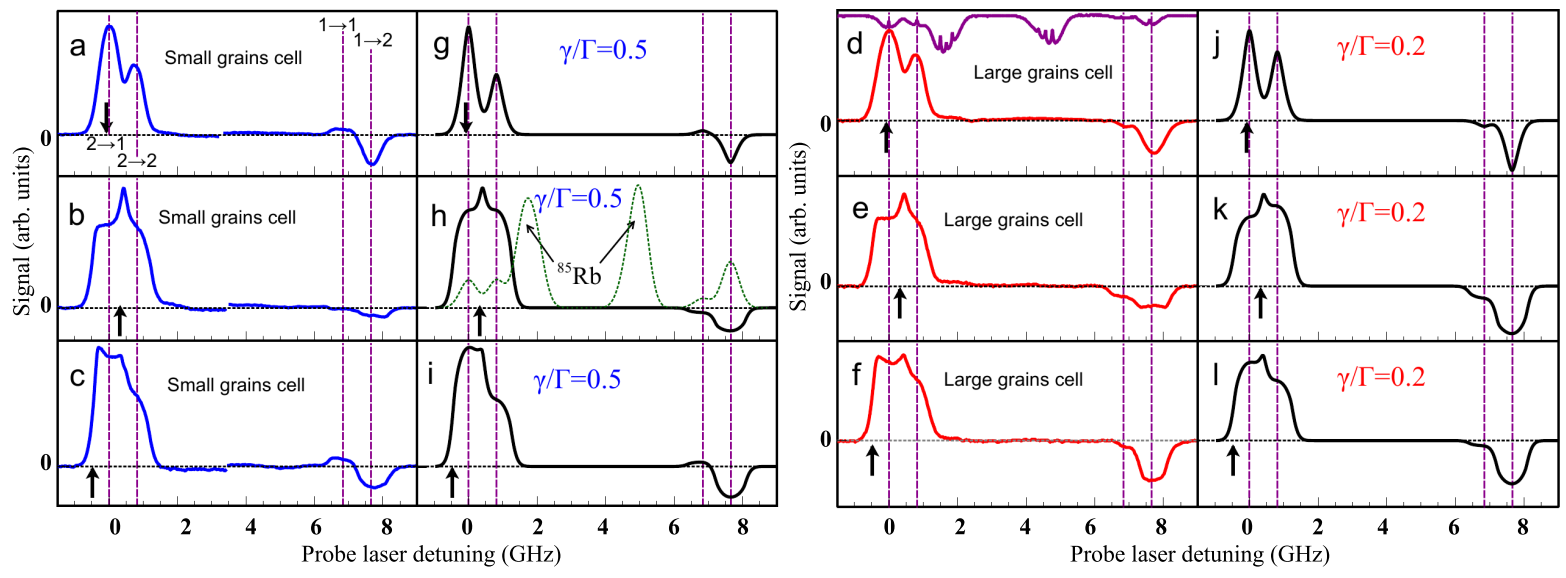

Fig. 2. (a-f) Experimental spectra measured in a $100 \mu \mathrm{m}$ cell (a-c) and in a $10 \mu \mathrm{m}$ cell (d-f) and the corresponding theoretical predictions after a simplified model (g-l). The pump frequency is indicated by the arrow. The dotted curve is a calculated linear response of the medium. An example of the SA spectrum used as a probe frequency reference is also shown at (d).

todiode was synchronically detected at the sum of modulation frequencies to extract the non-linear signal. At Fig.2 are shown the experimental measurements for different pump frequencies, for a $10 \mu \mathrm{m}$ pores cell (Fig.2(a-c)) and for a $100 \mu \mathrm{m}$ pores cell (Fig.2(d-f)). After a proper choise of the parameter $\gamma$, these measurements are well reproduced with the simplified model, including the relative peaks amplitudes and signs.

The possibility to observe coherent resonances in the transmitted light, i.e. with light fields with its amplitude, phase and propagation direction completely randomized is currently explored.

\section{References}

1. D. S. Wiersma, M. P. van Albada, and A. Lagendijk, Physical Review Letters 75, 1739 (1995).

2. Q. Baudouin, N. Mercadier, V. Guarrera, W. Guerin, and R. Kaiser, Nature Physics 9, 357 (2013).

3. M. Segev, Y. Silberberg, and D. N. Christodoulides, Nature Photonics 7, 197 (2013).

4. E. G. van Putten, D. Akbulut, J. Bertolotti, W. L. Vos, A. Lagendijk, and A. P. Mosk, Phys. Rev. Lett. 106, 193905 (2011).

5. O. Katz, E. Small, Y. Bromberg, and Y. Silberberg, Nature Photonics 5, 372 (2011).

6. I. M. Vellekoop, A. Lagendijk, and A. P. Mosk, Nature Photonics 4, 320 (2010).

7. B. Judkewitz, Y. M. Wang, R. Horstmeyer, A. Mathy, and C. Yang, Nature Photonics 7, 300 (2013).

8. T. Svensson, M. Andersson, L. Rippe, J. Johansson, S. Folstad, and S. Andersson-Engels, Optics Letters 33, 80 (2008).

9. T. Svensson and Z. Shen, Applied Physics Letters 96, 021107 (2010).

10. T. Svensson, E. Adolfsson, M. Lewander, C. T. Xu, and S. Svanberg, Physical Review Letters 107, 143901 (2011).

11. P. Ballin, E. Moufarej, I. Maurin, A. Laliotis, and D. Bloch, Appl. Phys. Lett. 102, 231115 (2013).

12. S. Villalba, H. Failache, A. Laliotis, L. Lenci, S. Barreiro and A. Lezama, Opt. Lett. 38, 193-195 (2013).

13. S. Villalba, A. Laliotis, L. Lenci, D. Bloch, A. Lezama and H. Failache, Phys. Rev. A 89, 023422 (2014).

14. S. Villalba, H. Failache, L. Lenci and A. Lezama, to be published. 